\title{
COMPARISON OF HEART RATE, GALVANIC SKIN REFLEX, PLETHYSMOGRAM, SUBJECTIVE FEELINGS AND PERFORMAMCE IN VIGILANCE AND ARITHMETICAL CALCULATION TASKS
}

\author{
Juichi KATO \\ National Institute of Industrial Health, Kizuki-Sumiyoshi, Kawasaki
}

(Received May 4, 1970)

Experiments were undertaken in order to compare changes of heart rate, galvanic skin reflex (GSR), photoelectric plethysmogram, subjective feelings and performance on a simple visual vigilance task (task I) with those on a continuous arithmetical calculation task (task II). Subjects were 10 male college students. Same subject performed the task I lasting for $1 \mathrm{hr}$ on the first day and the task II also for $1 \mathrm{hr}$ on the following day.

The increment of heart rate did not occur during the task I, but it occurred during the task II and the heart rate decreased after the task II. GSR frequency increased with the onset of both the tasks, giving a higher value during the task II than that during the task I and showing a decrease after both the tasks. The decreased amplitude of the pulse waves of plethysmogram did not appear at the beginning of the task I, whereas it had a tendency to appear at the same period of the task II.

Although the task I evoked mainly subjective feelings such as boredom and drowsiness, the task II evoked a strained state and a local fatigue of the right hand and arm. Subjective feelings after the task I were bored and drowsy as compared with those after the task II. Some subjects were in a slightly fatigued state and others were in a state of slightly refreshed frame of mind after the task II. Furthermore, they reported that the task II was easily performed as compared with the task I, though the task II seemed to be harder than the task I in relation to the changes of physiological functions.

There was no correlation between signal detection in the task I and correctness in the task II.

It has been found that increment of heart rate does not occur during a simple visual vigilance task ${ }^{1)}$, whereas accelerated level of heart rate occurs during a continued arithmetical calculation task ${ }^{2}$.

It is of interest to study the relation between the degree of acceleration of 


\section{J. KATO}

physiological functions and subjective feelings such as fatigue and boredom during and after these tasks.

Therefore, the present study was undertaken in order to compare the effects of a simple visual vigilance task and an arithmetical calculation task on heart rate, QSR, photoelectric plethysmogram and subjective feelings during and after both the tasks, and to investigate further the relation between performances in these tasks.

\section{Methods}

Subjects were ten healthy male students of 20-24 years old. Each subject came to the laboratory on two successive days. The subject rested in a sitting position during a period of $1 \mathrm{hr}$ before experiments. There were two experiments. Both the experiments consisted of a period of $1 \mathrm{hr}$ before the task (1.00-2.00 PM), a period of $1 \mathrm{hr}$ during the task (2.00-3.00 PM) and a period of $1 \mathrm{hr}$ after the task (3.00-4.00 PM), and were performed by one subject in a sitting position in a quiet room. The ambient temperature in the room during the experiments ranged from 22 to $24^{\circ} \mathrm{C}$. In experiment I a clock test which employed a rate of 35 signals/hr (task I) was used on the first day as a visual vigilance task in which the subject waited and responded to double jumps of a pointer on a clock panel. The apparatus for the task was the same as described previously ${ }^{1)}$. In experiment II Kraepelin-Uchida's test (task II) wan used as a continuous arithmetical calculation task.

Heart rate of the subject was measured by electrocardiogram with chest leads during the first three minutes of every ten minutes. GSRs were obtained from the electrodes attached to the left hand and frequencies in every ten minutes were used as GSR value. Photoelectric plethysmogram of the left index finger was recorded by using a reflexion photoelectric plethysmograph. All measurements were continuously recorded with a multichannel recorder throughout the experiments. After the experiments the subject was questioned about his subjective feelings before, during and after both the tasks. He was given neither knowledge of results nor reward on the performance of the task I or II.

\section{Results}

\section{Heart rate in experiments $I$ and $I I$}

The results are shown in Figs. 1, 2 and 3. In experiment I the heart rate for each subject before the task I ranged from 56.7 to 88.7 per min with the mean of 70.0. The increment of heart rate did not occur at the beginning of the task I. The mean value of heart rate during the task I was not significantly higher than that before the task I, though some showed slight increase as seen in subjects $2,3,5$ and 8. The heart rate for each subject during the task I ranged from 52.3 to 78.7 per min with the mean of 69.2 . The mean value after the task I decreased significantly as compared with that during the task $\mathrm{I}(\mathrm{P}<0.01)$. However, 


\section{COMPARISON OF VIGILANCE AND ARITHMETICAL CALCULATION TASKS}

one subject ( 8 ) showed a slight increase after the task I. The heart rate for each subject after the task I ranged from 52.0 to 77.3 per min with the mean of 63.5.

In experiment II the heart rate for each subject before the task II ranged from 55.0 to 89.3 per min; the mean was 72.4 . The heart rate increased at the beginning of the task II and this initial increment was maintained throughout the task. The mean value of heart rate during the task II was significantly higher than

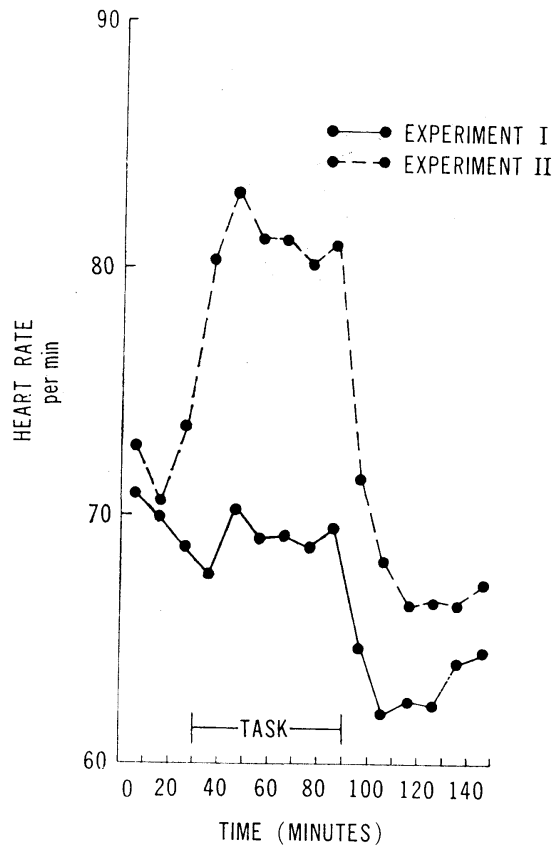

Fig. 1. Time course of mean heart rate obtained from the ten subjects in the experiments I and II.

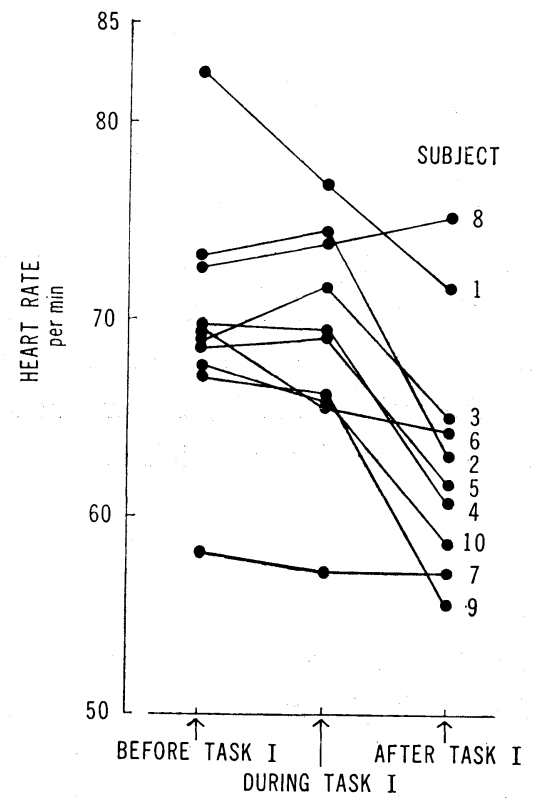

Fig. 2. Mean heart rate for each subject before, during and after the task I. 


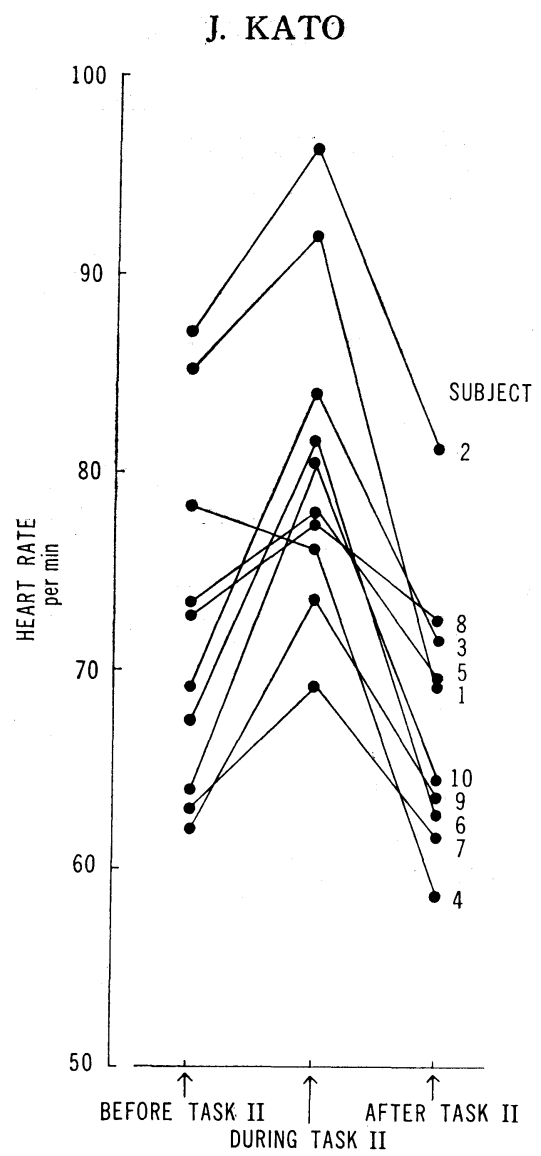

Fig. 3. Mean heart rate for each subject before, during and after the task II.

that before the task $(\mathrm{P}<0.01)$. However, one subject (4) did not show a increment of mean value with the onset of the task II. The heart rate for each subject during the task II ranged from 67.0 to 99.0 per min; the mean was 81.2. The mean value after the task II decreased significantly as compared with that during the task II $(\mathrm{P}<0.01)$. The heart rate for each subject after the task II ranged from 55.7 to 85.7 per min; the mean was 67.8 .

There was no significant difference between the mean values before the tasks I and II. The mean value during the task II was significantly higher than that during the task $\mathrm{I}(\mathrm{P}<0.01)$. Furthermore, there was no significant difference between the mean values after the tasks I and II, but in six subjects $(2,3,5,7,9,10)$ the mean values after the task II tended to be high as compared with those after the task I.

\section{GSR frequency in experiments $I$ and $I I$}

The results are shown in Figs. 4, 5 and 6 . In experiment I the initial increase of GSR frequency was found at the beginning of the task $I$ and it tended to decrease as the task progressed further, but in a few subjects this increment did not occur. GSR frequency for each subject before the task I ranged from 0 to 36 per $10 \mathrm{~min}$ 
with the mean of 4.5 . In three subjects $(2,8,9)$ GSR did not appear before the task I. In two subjects the GSR frequency increased at the last 10 -min period before the task I. The mean value of GSR frequency during the task I was significantly higher than that before the task $(P<0.01)$. The GSR frequency for each subject during the task I ranged from 0 to 72 per 10 min with the mean of 11.9. The difference between the mean values during and after the task $I$ was significant $(\mathrm{P}<0.01)$. The GSR frequency for each subject ranged from 0 to 28 per 10 min with the mean of 4.0. In one subject ( 8 ) the GSR did not appear throughout the experiment I.

In experiment II the initial increase was observed at the beginning of the task II and tended to decrease as the task progressed further, but in one subject' this increment did not occur. The GSR frequency for each subject before the task II ranged from 0 to 24 per $10 \mathrm{~min}$; the mean was 4.2 . In subjects $4,7,8$ and 9 the GSR did not appear before the task II. In two subjects the increment of GSR frequency was observed at the last 10 -min period before the beginning of the task II. The mean value of GSR frequency during the task II increased significantly $(\mathrm{P}<0.01)$. The GSR frequency for each subject during the task II ranged from 0 to 74 per $10 \mathrm{~min}$; the mean was 22.3. The difference between mean values during and after the task II was significant $(\mathrm{P}<0.01)$. In subjects 2,7 and 8 the mean values increased slightly after the task II.

There was no significant difference between the mean values before the tasks

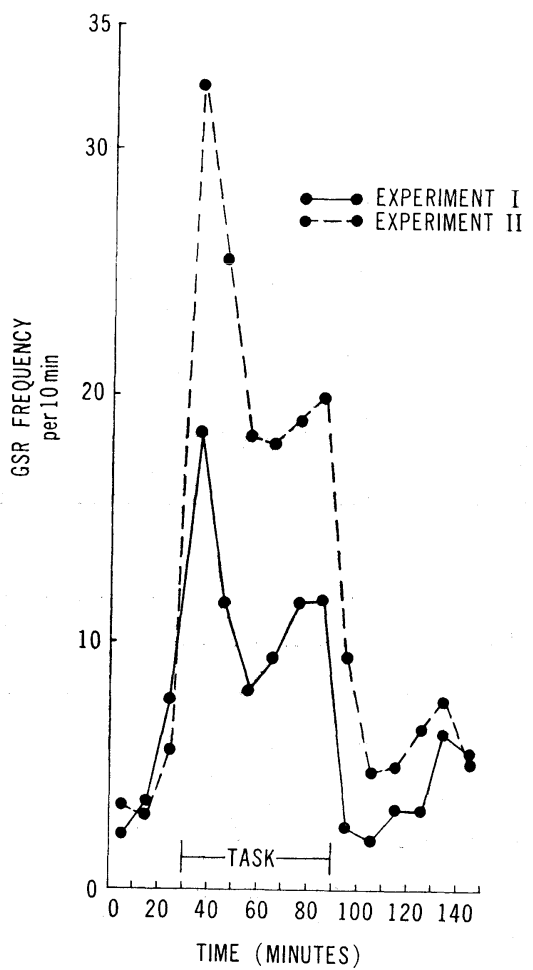

Fig. 4. Time course of mean GSR frequency obtained from the ten subjects in the experiments I and II. 


\section{J. KATO}

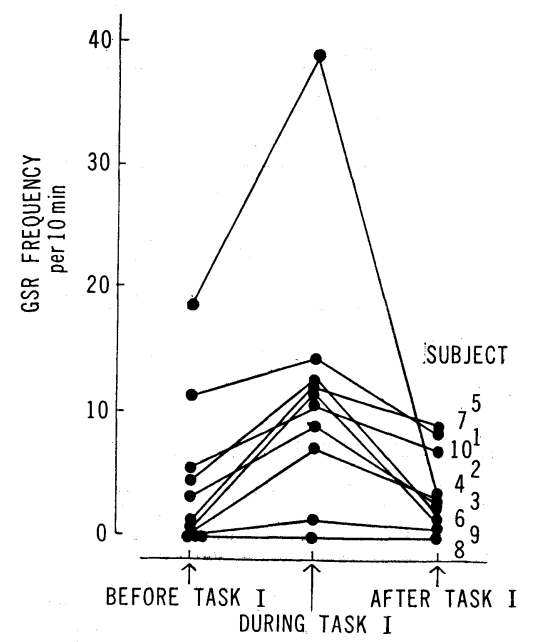

Fig. 5. Mean GSR frequency for each subject before, during and after the task I.

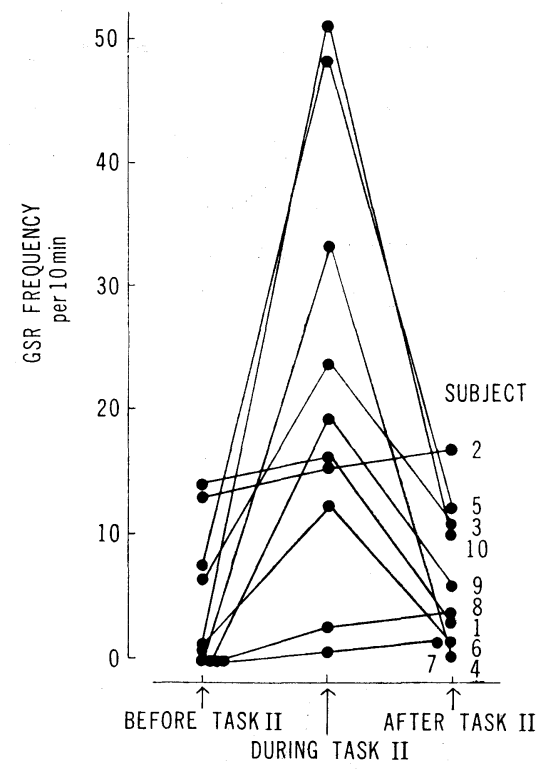

Fig. 6. Mean GSR frequency for each subject before, during and after the task II.

I and II. The mean value during the task II was significantly higher than that during the task I $(\mathrm{P}<0.05)$. Furthermore, there was no significant difference between the mean values after the tasks I and II. One subject ( 8 ) showed no GSR throughout the experiment I and slight one during and after the task II.

\section{Photoelectric plethysmogram in experiments I and II}

In experiment I the amplitude of the pulse waves of plethysmogram was similar before, during and after the task I, but only one subject showed the decrement of the amplitude at the beginning of the task.

In experiment II the amplitude of the pulse waves had a tendency to decrease 


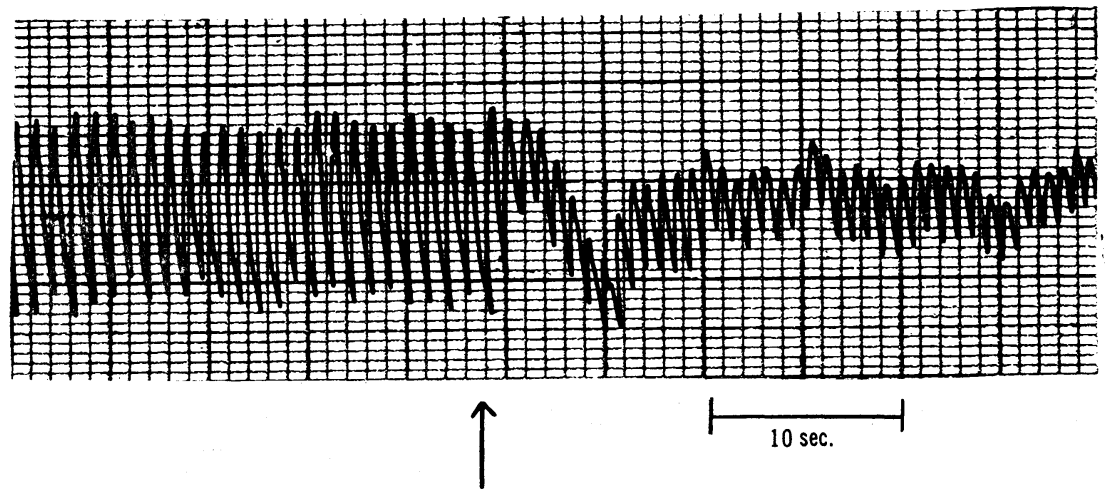

Fig. 7. A plethysmographic record from the left index finger of one? subject at the beginning of the task II. Arrow indicates that the subject started on the task.

at the beginning of the task II as seen in Fig. 7 and to increase after that peniod, but in some subjects this decrement of the amplitude was slight or did not appear clearly. One subject who had shown the decreased amplitude at the beginning of the task I had the same tendency in the same period of the task II.

\section{Relation between performances in tasks I and II}

There was no significant correlation between signal detection in the task I and correctness in the task II. The statistical correlation between signal detection in the task I and output in the task II was also not significant. Furthermore, the statistical correlation between correctness and output in the task II was not significant.

\section{Subjective feelings in experiments $I$ and $I I$}

During the task I the subjects felt a bored, relaxed, restless and drowsy state. During the task II they felt a strained state and a local fatigue of the right hand and arm. They reported that their subjective feelings after the task I were more bored, relaxed and drowsy as compared with that after the task II. Some subjects were in a slightly fatigued state and others were in a state of slightly refreshed frame of mind after the task II. Furthermore, all subjects reported that the task II was easily performed as compared with the task I and that they liked to perform the task II than the task I.

\section{Discussion}

From the data of the present study, it was found that the increment of heart rate did not occur during the task I, but in the experiment II heart rate increased during the task II and decreased after the task. Therefore, there was a difference between the heart rates during tasks I and II. On the effect of continued arithmetical calculation test on heart rate, we already found that heart rate in- 


\section{J. KATO}

creased immediately after the beginning of the test (Kraepelin-Uchidas test) and maintained in accelerated level during a certain period of time ${ }^{2}$. And also, it has been reported that heart rate does not increase during a 2 -hr period of a simple visual vigilance task which employs a low signal rate ${ }^{1)}$. These observations correspond with the result of the present study. Ishibashi et $\mathrm{al}^{3)}$. have reported that the more difficult a mental task is, the more increase is seen in heart rate and heart rate may be a useful method for estimation of mental load. Therefore, the task II is more harder than the task I in relation to the heart rate.

The GSR frequency increased with the onset of the task I or II, giving a higher value during the task II than that during the task I. This may mean that the state of emotional strain in relation to GSR frequency is higher during the task II than during the task I.

On the change of plethysmogram during the task I or II, it was found that the amplitude of the pulse waves had a tendency to decrease at the beginning of the task II, but it did not have such a tendency in the task I. The decrement of amplitude observed in this study corresponds with the result reported by $\mathrm{Na}$ gashima ${ }^{4)}$ who found it in mental calculation.

Baker and Ware ${ }^{5}$ have reported that vigilance performance is not predictable from performance of other monotonous tasks and a vigilance task appears to contain elements not found in other monotonous works. Although the present study investigated the relation between performance during the vigilance task and the arithmetical calculation task, there was no significant correlation between the signal detection in the task I and the correctness in the task II. Moreover, there were no significant correlation between the signal detection in the task I and the output in the task II. From these results, it seems that the performance of the task I may not be calculable from that of the task II.

The heart rate during the task I did not increase, whereas an increment of heart rate occurred with the onset of the task II. The GSR frequency increased with the onset of the task I or II, but the GSR frequency during the task II was higher than that during the task I. The decreased amplitude of the waves had a tendency to be observed at the beginning of the task II, but little changes occurred at the same period of the task I. In this way, the task I evoked little acceleration of the heart rate, little decrement of the amplitude and the increment of the GSR frequency which was lower than that during the task II, though the task II evoked clearly accelerations of these physiological functions. On the other hand, the subjects reported boredness and drowsiness during the task I but a strained state and local fatigue of the right hand and arm during the task II.

After the task II the heart rate decreased and there was no significant difference between heart rates after the tasks I and II. The increased GSR frequency during the task I or II decreased after both the tasks. The GSR frequency after the task II showed a similar value to that after the task I. However, the subjects reported that their subjective feelings were more bored, relaxed and drowsy after 


\section{COMPARISON OF VIGILANCE AND ARITHMETICAL CALCULATION TASKS}

the task I as compared with those after the task II, and a slightly fatigued state or slightly refreshed frame of mind after the task II.

It seems likely that one of the factors of the bored state may be due to little acceleration of heart rate and change of plethysmogram or low increment of GSR frequency during the task I and that one of the factors of the slightly fatigued state or the slightly refreshed frame of mind may be due to the acceleration of these physiological functions during the task II.

It is interesting to note that all subjects reported that the task II was easily performed as compared with the task I and that they liked to perform the task II, though the task II seemed to be harder than the task I in relation to the changes of these physiological functions. However, it must be noted that the subjects used in the present study were college students, because Kiriharab) has reported that female workers in industry like an experimental paced task, but investigators in a laboratory like an experimental unpaced task.

\section{AcKNowledgement}

The author expresses his thanks to Dr. H. Sakabe and Dr. S. Koshi in this institute for valuable suggestions, and to Mr. K. Nozaki in this institute for valuable helps and advices.

\section{REFERENCES}

1) Kato, J. (1968). Ind. Health, 6, 59.

2) Kato, J. Kojima, A. and Niiyama, Y. (1965). Ind. Health, 3, 1.

3) Ishibashi, T., Ohtani, A. and Miura, T. (1968). Japan. J. Ind. Health, 10, 377. (in Japanese).

4) Nagashima, C. (1956). Nippon Gekagakkai Zasshi, 57, 571 (in Japanese)

5) Baker, R. A. and Ware, J. R. (1966). Ergonomics, 9, 109.

6) Kirihara, S. H. (1960). J. Sci. Labour, 36, 621. (in Japanese) 